\title{
TERMINOLOGIA E DIVULGAZIONE DI TEMATICHE ECONOMICHE NELLA STAMPA ITALIANA
}

\author{
Elżbieta JAMROZIK \\ Università di Varsavia
}

\begin{abstract}
The aim of this research is to discuss the language of the Italian economic press: this linguistic variety can be analyzed as an LSP, with its particular type of terminology and lexical collocations, and as a journalistic text, with the lexical strategies proper to scientific dissemination. It is also important to mention the opposition between the scientific plan characterized by a specific terminology, loans and acronyms and the strategies used to transmit scientific content to a larger and less expert public. Making the text more accessible to the common user involves various forms of explanation, the use of metaphors and figured structures.
\end{abstract}

Si la parole est un des plus grands avantages de l'homme, ce ne doit pas estre une chose méprisable de posseder cet avantage avec toute la perfection qui convient à l'homme; qui est de n'en avoir pas seulement l'usage, mais d'en penetrer aussi les raisons, \& de faire par science, ce que les autres font seulement par coutume.

Grammaire générale et raisonnée de Port Royal (1660), Préface, p. 4

Ali, z podziękowaniem za stworzenie tylu mitych okazji do wspólnych-i jakże mitych! - refleksji nad językiem i nie tylko... 


\section{Premessa}

Scopo della presente disamina è mettere a confronto un tipo particolare di terminologia, quella economica, con le strategie lessicali proprie alla divulgazione scientifica. Il problema della terminologia economica nella stampa va affrontato nel quadro della distinzione tra linguaggio specialistico e linguaggio divulgativo (Sobrero, 1993: 241-2; Gualdo, Telve, 2011: 181-216). In ambedue i casi si tratta di varietà diafasiche sottostanti al contesto comunicativo, in cui l'utilizzo della terminologia, dei tratti testuali e delle strutture morfo-sintattiche tipiche rimane in stretta dipendenza con il destinatario del messaggio, lo scopo dell'atto comunicativo e l'argomento trattato. Ne consegue l'opposizione, nell'ambito del campo qui affrontato, ovvero la lingua dell'economia, tra

- il piano scientifico (dedicato agli specialisti del settore) caratterizzato da una forte presenza della terminologia monoreferenziale, da specifiche strutture morfo-sintattiche e testuali, neutralità emotiva e precisione semantica;

- il piano divulgativo atto a trasmettere dei contenuti scientifici ad un pubblico più vasto meno esperto (Sobrero, ibid., p. 241-242), il che implica un minore addensamento terminologico (termini sostituiti da pseudo-tecnicismi) e il ricorso a strategie di esplicitazione e spiegazione che mirano a rendere il testo più accessibile all'utente comune. Nella divulgazione dei contenuti un ruolo di primaria importanza spetta ai mass media (Gualdo, Telve, ibid., p. 188-192).

La scelta della lingua dell'economia come oggetto della disamina è stata dettata dal fatto che è proprio questo campo che gode odiernamente di un livello divulgativo particolarmente sviluppato. La stampa economica, nonché le pagine economiche dei grandi quotidiani hanno subito negli ultimi decenni un profondo cambiamento, in quanto l'economia coinvolge sempre più il pubblico dei lettori/ ascoltatori/telespettatori, a misura che strati sempre più ampi della popolazione si sono resi conto che il loro benessere privato è ormai inesorabilmente legato a fenomeni economici di natura globale. In conseguenza, da quando le questioni economiche costituiscono il campo di comune interesse di una fascia sempre più larga della società, si è accresciuta la domanda per l'informazione economica, potenziando a sua volta il volume dell'offerta giornalistica: in particolar modo la stampa, cercando di rispondere all'interesse di un numero crescente dei lettori, è passata da un ermetismo economico dedicato ai happy few a una lingua più amichevole, più comprensibile. Ne consegue il successo della lingua dell'economia a livello divulgativo in confronto alle altre lingue speciali, caratteristica rilevata già negli anni Novanta del secolo precedente da Scavuzzo (1992: 173) e Sobrero (op. cit., p. 256-259). 


\section{Metodologia e corpus}

Rispetto alla varietà speciale le cui caratteristiche sono state evidenziate sopra rispetto al common core della lingua, la varietà divulgativa manifesta un netto intento perlocutivo realizzato tramite aggettivazioni, metafore, eufemismi, pseudotecnicismi, il calo della precisione. All'interno dei testi del settore economico risulta alta anche la presenza di strutture fisse o semi-fisse (collocazioni) e di anglicismi, specie non adattati, sia nella terminologia stessa che nell'insieme del testo.

Ci siamo proposti di mettere a confronto i mezzi lessicali e le strategie ai quali ricorre la stampa italiana presentando la problematica della crisi economica, al fine di:

1) individuarvi le caratteristiche proprie alla varietà speciale rispetto a quelle della divulgazione;

2) stabilire il grado di saturazione del testo con elementi specifici per la varietà speciale in un confronto quantitativo (Cortelazzo, 2013);

3) valutare la stabilità delle strategie proprie per la lingua divulgativa della tematica della crisi qui considerata; a questo fine viene realizzato il confronto di due corpora-campione appartenenti a periodi temporali distinti.

Ci concentreremo sull'aspetto lessicale della varietà speciale, ovvero la presenza di termini specifici e collaterali (Serianni, 2003: 81-83), i meccanismi di costruzione e la struttura dei termini specifici, nonché le combinazioni lessicali in cui essi riccorrono. Per la varietà divulgativa verranno considerati:

- i mezzi lessicali sostitutivi della terminologia o complementari rispetto ad essa;

- le strategie testuali che mirano a facilitare al lettore comune la comprensione della problematica specialistica (metafora, paragone);

- le collocazioni e i fraseologismi che incidono sulla rigidità della lingua dell'economia, ma nel contempo, per la loro alta frequenza (ripetitività) ne agevolano la comprensione.

Il nostro studio è basato su due corpora-campione tratti da "Il Sole 24" ore, quotidiano riconosciuto e affermato nel settore economico-finanziario (Gualdo, Telve, op. cit., p. 367): il primo comprende testi del 2013-14 e si focalizza sulla crisi economica in Grecia, il secondo - testi del 2017-18, sempre accentrati sulla tematica della crisi, tuttavia in un'ottica più generale. La scelta di tematiche affini ma per certi versi distinte mirava a verificare se la natura dell'argomento trattato incideva o meno sul tipo di lingua usata nel testo.

Corpus 1: Limiti cronologici: 01.03.2013-01.03.2014; Lunghezza - 32.476 parole. 
Corpus 2: 13.01.2017-11.01.2018; 38.919 parole.

Nel quadro dell'analisi lessicale ci siamo in parte avvalsi della teoria dell'immagine linguistica del mondo, approccio che, continuando il filone filosofico-linguistico sorto in Germania (la innere Sprachform di J. G. Herder, la Weltanschaung di W. von Humboldt, la lingua come specchio del pensiero di Vossler, Sprache - Schlüssel zur Welt di H. Gipper) negli ultimi decenni è stato approfondito da linguisti slavi (Anusiewicz, 1999). In questo approccio la lingua diventa fonte di informazioni su se stessa in quanto il suo uso consiste a categorizzare, strutturare e interpretare la realtà. L'immagine linguistica del mondo di una comunità parlante si rivela attraverso le proprietà grammaticali della sua lingua (flessione, morfologia derivativa, strutture sintattiche), ma soprattutto nella struttura semantica del lessico che riflette la percezione e la valorizzazione degli elementi della realtà da parte dei parlanti (Grzegorczykowa, 1993; Bartmiński, 2008, 2009). Infatti i fenomeni lessicali classificano il mondo tramite l'organizzazione della struttura semantica delle parole, il loro significato denotativo e connotativo, nonché le relazioni che intrattengono con altri lessemi. In questo senso le scelte linguistiche operate dagli autori dei testi del corpus possono essere almeno in parte interpretate in chiave della loro percezione individuale dei fenomeni economici trattati.

\section{Terminologia}

Le caratteristiche della terminologia economica sono state ampiamente studiate sotto vari aspetti (Gualdo, Telve, op. cit., p. 361-374): quello storico seguiva la nascita e il progressivo sviluppo dei termini vernacolari che, sorti dall'attività dei mercanti fiorentini, veneziani, genovesi, oggidì costituiscono il nucleo della disciplina (Sosnowski, 2006: 26-29), quello degli influssi stranieri, prima francesi (Librandi, 1997; Dardi, 1992) e in seguito inglesi - prestiti adattati e calchi la cui origine si è ormai persa nella coscienza degli utenti, fino agli onnipresenti prestiti non-adattati angloamericani dei tempi moderni. Il corpus studiato abbonda necessariamente di rappresentanti delle categorie soprannominate, che siano lessemi semplici o unità create per derivazione, formazioni denominali (creditore, debitore) o sostantivi deverbali (recessione, stagnazione, importazione) la cui relazione con il lessema di base diventa occultata in modo da funzionare come unità semantiche autonome.

Particolarmente rappresentate sono le polirematiche (Scarpa, 2002: 31-32), già segnalate da Librandi (ibid., p. 249) per la terminologia dei secoli precedenti, di struttura Determinato-Determinante: 
- N + Agg: settore pubblico/privato, debito pubblico, valore aggiunto;

$\bullet \mathrm{N}+\mathrm{SP}$ : tasso d'interesse.

Tra questi tecnicismi, di diffusione ormai così ampia da fondersi quasi con il lessico commune, spiccano ogni tanto elementi stranieri:

- neologismi: Euroland, eurogruppo, eurozona, (scongiurare) l'eurocrac;

- prestiti integrali, per lo più angloamericani: (rating, bailout), tra cui anche quelli prettamente stilistici (problem-solving, il peso dell'export);

- rari prestiti da altre lingue: troika, tranche (la nuova tranche di aiuti);

- prestiti combinati, ovvero strutture polirematiche anglo-italiane o italoinglesi: accordi di repurchase, il gap fiscale.

Ad accrescere le difficoltà di comprensione contribuiscono non poco sigle ed acronimi, opachi a chi non è familiarizzato con il settore: BCE (Banca Centrale Europea), FMI (Fondo Monetario Internazionale), MES (Meccanismo Europeo di Stabilità).

\section{Collocazioni}

Nella comunicazione speciale un posto di riguardo spetta alle collocazioni, combinazioni di lessemi che, senza essere ancora fisse, spiccano per lo meno per la loro frequenza d'uso, come testimoniato dalle ricerche svolte sui corpora. Lo stile specifico per una disciplina scientifica dipende in gran parte da fattori distribuzionali (Serianni, 2003: 81), per cui sia la didattica delle lingue speciali, in particolar modo quella indirizzata agli stranieri (Jamrozik, 2014; Kaliska, 2012), sia la didattica della traduzione, dovrebbero conferire un'importanza particolare a quelle combinazioni lessicali, definite privilegiate da J. R. Firth (1957: 11-12) che fu tra i primi a adoperare il termine di collocation. Specie in ottica contrastiva, bilingue, risulta importante conoscere le combinazioni privilegiate, al fine di evitare le interferenze e saper scegliere il lessema adeguato, proprio, invece di quello generico (fare $>$ applicare le riforme; il deficit/il debito è di... > ammonta a...). Infatti a realizzare un testo scientifico riuscito contribuisce non solo la scelta dei termini, ma anche la selezione, tra le varie unità apparentemente sinonimiche, di quelle che accompagnano il termine in modo più felice rispetto ad altre. Di queste scelte testimoniano sia i dizionari (Lo Cascio 2012; Tiberii 2012), sia le ricerche sui corpora (Cresti, Panunzi, 2013: 126-140) che rilevano le combinazioni più frequenti.

Considerando il lessema crisi nella sua accezione economica, il dizionario (Tiberii, ibid., p. 153) elenca una serie di aggettivi provvisti, con la sola eccezione di leggera e breve, di una componente semantica negativa di grado di intensità variabile (acuta, difficile, drammatica, duratura, grave, inevitabile, intensa, 
leggera, perenne, permanente, profonda, ricorrente, seria, terribile, violenta); per quanto riguarda le combinazioni verbali, crisi è soggetto di: si attenua, ha ripercussioni su, esplode, si profila, si protrae, sconvolge, scoppia, travolge. Invece in posizione di oggetto il lessema accompagna verbi come: affrontare, aggravare, attraversare (un periodo di), controllare, essere sull'orlo di, gestire, prevenire, provocare, risolvere, scatenare, scongiurare, superare, tamponare uscire da. Confrontando i dati lessicografici con quelli del corpus raccolto risulta che i giornalisti sfruttano ampiamente le combinazioni riportate dai dizionari come più frequenti; nei testi del corpus ritroviamo infatti, rimanendo nello stretto campo dell'economia, le combinazioni seguenti:

- $\mathrm{N}+$ Determinante (Agg/SP): crisi del mercato, crisi aziendale, crisi finanziaria, crisi bancaria, crisi europea, crisi del debito, crisi senza sbocchi e anche strutture elittiche come crisi spagnola/greca, crisi subprime;

- Nsogg. + V: crisi finita, la crisi sconvolge i mercati, la crisi travolge il mercato orafo;

- V + N compl.: uscire dalla crisi, affrontare la crisi, superare la crisi, resistere alla crisi, portare il Paese fuori dalla crisi, la crisi colpisce al Sud.

Va notato tuttavia che sono stati rilevati anche vari casi di creatività dei giornalisti, in accordo con le esigenze del medium e dello stile brillante adoperatovi, che oltrepassano per l'intensità e l'immaginario le metafore insite nella terminologia (Prandi, 2013: 8, 36) e le combinazioni lessicali usuali: famiglie massacrate dalla crisi, paese stritolato dalla crisi, la crisi manda in tilt le aspettative.

\section{Strategie di divulgazione}

Oltre a rilevare la terminologia, il corpus raccolto va studiato anche sotto il profilo delle strategie divulgative adoperatevi, nonché dal punto di vista dei procedimenti stilistici propri al giornalismo e ai mass media che portano alla detta "mediamorfosi" delle notizie (Antonelli, 2007: 93).

\subsection{SPIEgAZIONE}

Le strategie di divulgazione consistono soprattutto a ridurre il distacco tra l'utente comune e il carattere specialistico del testo, permettendo al lettore di non sentirsi escluso, per via della propria ignoranza nel settore, dalla tematica trattata. Il 
mezzo fondamentale ne è la spiegazione esplicita (ovvero, cioè) o meno esplicita che riguarda:

- i termini: ...l'incontro dell'eurogruppo, ovvero dei ministri delle finanze della zona euro...;

- le sigle e gli acronimi: ...gestiti dalla BCE (Banca Centrale Europea)...;

- i prestiti integrali: .... il "haircut" deciso dall'UE, tagli a scapito di creditori privati, doveva contribuire ad abbassare il debito...; ...le risoluzioni della troika, cioè degli esperti congiunti della Commissione Europea, della Banca Centrale Europea e del Fondo Monetario Internazionale.

Per quanto riguarda i prestiti integrali dall'inglese nel lessico della stampa, se il loro numero sale in modo vertiginoso tra gli anni ' 60 e '90 del Nocevento (Gualdo, Telve, 2011: 368), testimonianza di cambiamenti economici e sociali, ma anche di evoluzione nelle mentalità, per l'argomento qui studiato esso dipende dalla tematica del testo e dal grado della sua tecnicità, nonché dal profilo del supposto lettore. Da questo punto di vista gli articoli raccolti non sono omogenei: alcuni sono più divulgativi, altri risultano altamente specialistici, il che si manifesta tra l'altro nella saturazione con prestiti integrali e sigle.

Per rendere conto di questa differenza abbiamo esaminato due articoli del corpus di lunghezza comparabile (1000 parole circa): il primo portava sulle azioni finanziarie specifiche messe in atto durante la crisi greca (Paolo Becchi, Meccanismo Europeo di Stabilità: tutto quello che non vi dicono e che dovreste sapere, 03.04.2014), il secondo valutava in modo molto più generico la situazione mondiale e italiana nel periodo successivo alla crisi (Paolo Bricco, A 10 anni dalla crisi il mondo è ripartito, l'Italia resta in affanno, 13.01.2017).

Testo 1:

Prestiti integrali: 5 lessemi con 8 occorrenze di cui:

- 2 prestiti di termini specifici: hedge (1), repurchase (1)

- 3 prestiti del linguaggio giornalistico: governance (3), welfare (2), mainstream (1)

Sigle: 8 sigle con 21 occorrenze, di cui:

- 2 sciolte: MES - Meccanismo Europeo di Stabilità (8); MOU - "cosiddetti protocolli d'intesa" (2)

- 6 non sciolte: IVA, Pil, Ocse, BCE (3), UE (2), FMI (3)

Testo 2:

Prestiti integrali: 4 lessemi di 1 occorrenza di cui:

- 4 termini specifici: "assembled", "designed”; export, export-oriented

- 2 citazioni non tradotte: "back to manufacturing”, "make America great again" 
Sigle: 4 sigle con 7 occorrenze, di cui:

- 2 sciolte: Banca Mondiale, Fondo Monetario Internazionale

- 2 non sciolte: Pil (5), Ocse (2).

Come risulta, la saturazione del testo con sigle non sciolte o prestiti integrali dipende dal tipo di articolo e dalla tematica trattata; va notato tuttavia che il numero globale dei termini inglesi opachi al lettore non è particolarmente alto: solo due nel testo 1 (hedge, repurchase); nel testo 2 i primi termini ("assembled", "designed") sono successivamente spiegati, gli altri due - export, export-oriented - sono internazionalismi trasparenti all'utente italiano; per quanto riguarda le numerose sigle del testo 1 , esse vengono sciolte alla prima occorrenza per apparire come sigle in seguito; quelle non sciolte invece sono considerate note al lettore colto del quotidiano. Ne consegue che le strategie divulgative che tendono ad abbassare il livello di complessità dei testi specialistici variano anche a seconda delle tematiche trattate; inoltre la presente sommaria ricerca comprova in parte il supposto profilo del lettore colto (Kaliska, 2014: 237).

\subsection{IMMAGINE}

Un'altra strategia che mira lo stesso scopo ma realizzato con mezzi diversi, è il ricorso all'immagine, alla metafora che attrae il lettore evitandogli la monotonia di un testo specialistico e facendogli percepire nel contempo un fenomeno economico astratto in modo concreto, quasi iconico, vicino alla realtà materiale, il che agevola la comprensione (Prandi, op. cit., p. 32-34). Questa strategia, presente nel giornalismo italiano ormai da decenni e definita come "stile brillante" (Bonomi, 2003: 158), nel settore qui considerato mira non solo a meravigliare, bensì a avvicinare tramite l'immagine. Alcune tra le immagini sono ormai fisse, stabilizzate nel dizionario, come quella della crisi che, quasi un proiettile, esplode e colpisce, altre si rivelano più innovative e rilevano dall'immaginario collettivo che il giornalista mette a profitto. Così la stessa crisi viene percepita come:

- un liquido, fiume o mare: la crisi si è riversata nella zona euro, la crisi dilaga, paese immerso nella crisi/nella recessione, il memorandum della troika concede liquidità a un paese in apnea; i contribuenti stanno annegando nei debiti fiscali;

- un vuoto, buco o addirittura voragine: un buco si apre nelle finanze/nei conti, un'altra voragine nei Conti ellenici, prestiti che non sanano il buco strutturale, tappare il buco, il paese sprofonda nella recessione, coprire il gap fiscale; 
- una malattia, per cui si ricorre alla terminologia del settore medico: frodi fiscali a carattere endemico, l'amministrazione cresciuta in modo abnorme, il settore pubblico roso dalla corruzione, risanamento dei conti.

Gli effetti di questa situazione economica sono profondamente negativi (effetti devastanti della crisi), e incidono sullo sviluppo che viene sminuito attraverso scelte lessicali appropriate: $i$ consumi si restringono, il reddito dei proprietari si è contratto, i redditi cadono/precipitano, l'inflazione frena i consumi.

Siccome l'economia è una scienza basata su leggi e disciplina rigorosa, le cause della crisi sono da ricercare nell' allentamento del rigore dovuto a: rendiconti creativi, contabilità creativa, derive fiscali, nonché nella disonestà di alcuni cittadini (frode fiscale, evasione fiscale).

Di conseguenza, per uscire da questo stato di cose occorre riapplicare rigore e disciplina tramite: tagli profondi, misure di austerità, una nuova tornata di austerità; con lo scopo di: rimettere sul binario giusto l'amministrazione pubblica; tenere il paese sulla corda per evitare eventuali derive, realizzare fino in fondo le riforme necessarie.

Inoltre occorre eliminare le cause nefaste di cui prima: fare la guerra alla frode fiscale, ridurre l'evasione fiscale, estirpare la burocrazia.

Tale situazione economica implica comunque sofferenza della popolazione, come palese specie nei testi dedicati alla crisi greca dove questo riferimento appare:

- in modo diretto tramite lessemi che denotano esplicitamente il dolore: tagli dolorosi per la società, portare il peso delle difficili riforme, Il popolo greco non ce la fa più, piani lacrime e sangue imposti al Paese, manovra lacrime e sangue, li aspetta ancora tanto dolore e lacrime, un paese stremato dai sacrifici e tagli, opinione pubblica esasperata; L'esasperazione della popolazione;

- indirettamente in scelte lessicali che implicano la sofferenza: Atene nella morsa dei creditori, Atene con i creditori alla porta; lo spettro di nuovi tagli; le multinazionali spremono i cittadini, un drammatico stringersi la cinghia.

Come risulta da questa breve disamina, nonostante il rigore insito in una disciplina come l'economia, i testi giornalistici del settore economico portano, benché in misura disuguale, l'impronta del medium che li veicola ricorrendo volentieri alla metafora creativa (tenere il paese sulla corda) o la citazione storica (lacrime e sangue). Da un'analisi sommaria, che andrebbe certamente affinata e completata da un'ingadine quantitativa, risulta che questa parte cresce notevolmente nelle tematiche che coinvolgono direttamente l'essere umano che subisce le conseguenze di un travolgimento economico, tematiche in cui il giornalista si sente direttamente coinvolto, si implica, almeno attraverso le scelte lessicali. 


\section{Conclusione}

La ricerca svolta su due corpora-campione di testi dell'ambito economico della crisi dimostra una relativa stabilità lessicale della terminologia e delle collocazioni usuali. Le procedure divulgative (spiegazioni, scioglimento delle sigle), rilevate nell'insieme del corpus, sono applicate con una frequenza che dipende dalla tematica trattata. Nel contempo si è notato un arricchimento a livello delle metafore e immagini, più creative nei testi di tematica meno spersonalizzata e oggettiva che presentano le implicazioni della situazione economica sulla popolazione e coinvolgono maggiormente l'autore-giornalista.

La presente ricerca potrebbe essere allargata successivamente in una dimensione contrastiva con il polacco. L'analisi contrastiva svolta su corpora paralleli, visto che generalmente non si dispone di traduzioni ufficiali dei testi dei quotidiani, potrebbe far emergere sia le corrispondenze che le divergenze tra le lingue nell'ambito lessicale e testuale, rivelando anche delle somiglianze e dissimilitudini tra la stampa italiana e polacca per quanto riguarda il modo di presentare informazioni e problemi analoghi.

\section{Riferimenti bibliografici}

ANTONELLI, Giuseppe (2007), L'italiano nella società della comunicazione, Bologna, Il Mulino. ANUSIEWICZ, Janusz (1999), « Problematyka językowego obrazu świata w poglądach niektórych językoznawców i filozofów niemieckich XX wieku », in Językowy obraz świata (J. Bartmiński éd.), Lublin, Wydawnictwo UMCS, pp. 261-289.

ARNAULD, Antoine, LANCELOT, Claude (1660), Grammaire générale et raisonnée de Port Royal, Paris, Pierre le Petit.

BARTMIŃSKI, Jerzy (2008), «Etnolingwistyka, lingwistyka kulturowa, lingwistyka antropologiczna?», Język a Kultura, t. 20, pp. 15-33.

BARTMIŃSKI, Jerzy (2009), Językowe podstawy obrazu świata, Lublin, Wydawnictwo UMCS.

BONOMI, Ilaria (2003), « La lingua dei quotidiani », in La lingua italiana e i mass media (I. Bonomi, A. Masini, S. Morgana éds), Roma, Carocci, pp. 127-164.

CORTELAZZO, Michele (2013), "Metodi qualitativi e quantitativi di analisi dei testi », Contemporanea, $\mathrm{n}^{\circ}$ 2, pp. 299-310.

CRESTI, Emanuela, PANUNZI, Alessandro (2013), Introduzione ai corpora dell'italiano, Bologna, Il Mulino.

DARDI, Andrea (1992), Dalla provincia all'Europa. L'influsso del francese sull'italiano tra il 1650 e il 1715, Firenze, Le Lettere.

FIRTH, John R. (1957), Papers in linguistics, London, Oxford University Press.

GRZEGORCZYKOWA, Renata (1993), « Teoretyczne i metodologiczne problemy semantyki w perspektywie tzw. kognitywnej teorii języka », in Studia semantyczne (R. Grzegorczykowa, Z. Zaron éds), Wydawnictwa Uniwersytetu Warszawskiego, Warszawa, pp. 9-22. 
GUALDO, Riccardo, TELVE, Stefano (2011), Linguaggi specialistici dell'italiano, Roma, Carocci. JAMROZIK, Elżbieta (2014), « Il lessico e le collocazioni nell'insegnamento delle lingue speciali », Kwartalnik Neofilologiczny, vol. LXI, n ${ }^{\circ}$ 1, pp. 163-176.

KALISKA, Marta (2012), « Teksty prasowe w nauczaniu włoskiego języka ekonomii », Lingwistyka stosowana, $\mathrm{n}^{\circ}$ 6, pp. 83-98.

KALISKA, Marta (2014), « L'italiano dell'economia di fronte alla supremazia dell'inglese », Studia romanica et anglica zagrabiensia, t. 5, pp. 231-238.

LIBRANDI, Rita (1997), « Sul lessico dell'economia negli scritti di Antonio Genovesi e Ferdinando Galiani », in Letteratura e industria (G. Bàrberi Squarotti, C. Ossola éds), vol. 1, Firenze, Olschki, pp. 239-252.

LO CASCIO, Vincenzo (éd.) (2012), Dizionario combinatorio compatto italiano, Amsterdam/ Philadelphia, John Benjamins.

PRANDI, Michele (2013), « Le metafore nella creazione di terminologia: una tipologia ragionata », in Il ruolo della metafora nella creazione di terminologie (M. Prandi, A. Giaufret, M. Rossi éds), Genova, De Ferrari, pp. 25-39.

SCARPA, Federica (2002), «Terminologia e lingue speciali », in Manuale di terminologia. Aspetti teorici, metodologici e applicativi (M. Magris, M. T. Musacchio, L. Rega, F. Scarpa éds), Milano, Hoepli, pp. 27-47.

SCAVUZZO, Carmelo (1992), " Il linguaggio delle pagine economiche », in Il linguaggio del giornalismo (M. Medici, D. Proietti éds), Milano, Montblanc, pp. 173-189.

SERIANNI, Luca (2003), Italiani scritti, Bologna, Il Mulino.

SOBRERO, Alberto (1993), «Lingue speciali », in Introduzione all'italiano contemporaneo. La variazione e gli usi (A. Sobrero éd.), Bari, Laterza, pp. 237-277.

SOSNOWSKI, Roman (2006), Origini della lingua dell'economia in Italia. Dal XIII al XVI secolo, Milano, Franco Angeli.

TIBERII, Paola (2012), Dizionario delle collocazioni, Bologna, Zanichelli. 